\title{
Avaliação da aprendizagem de enfermeiros em um curso online sobre úlcera venosa
}

\author{
Evaluation of nurses' learning in an online course about venous leg ulcer
}

Evaluación del aprendizaje de enfermeros en un curso online sobre úlcera venosa

\begin{abstract}
Fernanda Mateus Queiroz Schmidt',2, Juscilynne Barros da Costa Aroldi3, Heloisa Helena Ciqueto Peres ${ }^{4}$, Lilian Mara Quiroz ${ }^{5}$, Patrícia Pereira dos Anjos 6 , Vivian Aparecida Teixeira Vera Lúcia Conceição Gouveia Santos²
\end{abstract}

ORCID IDs

Schmidt FMQ (iD https://orcid.org/0000-0002-2454-6548

Aroldi JB da C (iD https://orcid.org/0000-0001-8565-0317

Peres HHC (iD https://orcid.org/0000-0002-8759-5670

Quiroz LM (iD https://orcid.org/0000-0002-4872-306X

dos Anjos PP (iD https://orcid.org/0000-0002-1356-9410

Teixeira VA (iD https://orcid.org/0000-0002-5864-4904

Santos VLCG (iD https://orcid.org/0000-0002-1288-5761

\section{COMO CITAR}

Schmidt FMQ, Aroldi JB da C, Peres HHC, Quiroz LM, dos Anjos PP, Teixeira VA, Santos VLCG. Avaliação da aprendizagem de enfermeiros em um curso online sobre úlcera venosa. ESTIMA, Braz.J. Enterostomal Ther., 16: e3718. https://doi.org/10.30886/estima.v16.613 PT

\section{RESUMO}

Objetivo: Avaliar o grau de conhecimento de enfermeiros sobre úlcera venosa (UV) e terapia compressiva (TC); comparar o grau de conhecimento sobre a temática entre enfermeiros com e sem formação especializada em estomaterapia, após a realização de curso online; e analisar as variáveis demográficas e educacionais associadas à aprendizagem. Métodos: Estudo quase experimental e comparativo em que se testou uma intervenção didática em dois grupos distintos de enfermeiros, por meio da aplicação de questionário para avaliação da aprendizagem antes e após o curso. Os dados foram analisados por testes McNemar, qui-quadrado, F da ANOVA, $t$ de Student e equações de estimação generalizada. Resultados: Os enfermeiros especialistas obtiveram notas médias 7,79 e 9,07 e os generalistas 6,39 e 8,49, respectivamente, nos pré- e pós-curso. A idade igual ou superior a 30 anos influenciou para o maior grau de conhecimento após o curso. Conclusão: O curso permitiu a aprendizagem sobre a temática em ambos os grupos. Enfermeiros especializandos tinham maior conhecimento sobre o tema quando comparados aos enfermeiros generalistas.

DESCRITORES: Estomaterapia; Úlcera varicosa; Bandagens compressivas; Informática em enfermagem; Educação a distância; Aprendizagem; Estomaterapia.

\footnotetext{
'Instituto Federal de Educação, Ciência e Tecnologia do Sul de Minas Gerais - Departamento de Enfermagem - Passos/MG - Brasil. 2Universidade de São Paulo - Escola de Enfermagem - Departamento de Enfermagem Médico-Cirúrgica - São Paulo/SP - Brasil. ${ }^{3}$ Exército Brasileiro - Arsenal de Guerra de São Paulo - São Paulo/SP - Brasil

${ }^{4}$ Universidade de São Paulo - Escola de Enfermagem - Departamento de Orientação Profissional - São Paulo/SP - Brasil.

IInstituto Brasileiro de Controle do Câncer - Serviço de Estomaterapia - São Paulo/SP - Brasil.

${ }^{6}$ A. C. Camargo Cancer Center - Ambulatório de Curativos - São Paulo/SP - Brasil.

${ }^{7}$ Hospital Cruz Azul de São Paulo - Clínica Cirúrgica - São Paulo/SP - Brasil.

Autor correspondente: Fernanda Mateus Queiroz Schmidt | Rua Santa Casa, 226, Sala 7 - Santa Casa | CEP: $37904-020$ - Passos/ MG - Brasil | E-mail: fernandamqueiroz@yahoo.com.br

Recebido: Jun 082018 | Aceito: Nov 212018
} 


\begin{abstract}
Objective: To evaluate the degree of knowledge of nurses about venous leg ulcer (VLU) and compressive therapy (CT); comparing the degree of knowledge on the subject between nurses with and without specialized training in stomatherapy, after the online course; and analyze the demographic and educational variables associated with learning. Methods: A quasi-experimental and comparative study in which a didactic intervention was tested in two different groups of nurses, through the application of a questionnaire to evaluate learning before and after the course. Data were analyzed by McNemar, chi-square, F of ANOVA tests, Student $t$ and generalized estimation equations. Results: The specialist nurses obtained average scores of 7.79 and 9.07 and the generalists of 6.39 and 8.49 , respectively, in the pre and post-course. Age equal or higher to 30 years influenced to the highest degree of knowledge after the course. Conclusion: The course allowed learning about the theme in both groups. Specialist nurses had greater knowledge on the subject when compared to generalist nurses.
\end{abstract}

DESCRIPTORS: Stomatherapy; Varicose ulcer; Compressive bandages; Computing in nursing; Correspondence course; Learning; Stomatherapy.

\title{
RESUMEN
}

Objetivo: Evaluar el grado de conocimiento de enfermeros sobre úlcera venosa (UV) y terapia compresiva (TC); comparar el grado de conocimiento sobre la temática entre enfermeros con y sin formación especializada en estomaterapia, después de la realización del curso online; y analizar las variables demográficas y educativas asociadas al aprendizaje. Métodos: Estudio cuasi experimental y comparativo en el que se probó una intervención didáctica en dos grupos distintos de enfermeros, por medio de la aplicación de un cuestionario para evaluación del aprendizaje antes y después del curso. Los datos fueron analizados por pruebas McNemar, x-cuadrado, F de ANOVA, $t$ de Student y ecuaciones de estimación generalizada. Resultados: Los enfermeros especializados obtuvieron notas promedios 7,79 y 9,07 y los clínicos 6,39 y 8,49, respectivamente, en los pre y post curso. La edad igual o superior a 30 años influyó para un mayor grado de conocimiento después del curso. Conclusión: El curso permitió el aprendizaje sobre la temática en ambos grupos. Enfermeros especializados tenían mayor conocimiento sobre el tema en comparación con los enfermeros clínicos.

PALABRAS CLAVE: Estomaterapia; Úlcera varicosa; Vendajes compresivos; Informática en enfermería; Educación a distancia; Aprendizaje; Estomaterapia.

\section{INTRODUÇÃO}

$\mathrm{Na}$ era do conhecimento, a velocidade das inovações, unida à exigência do mercado de trabalho por profissionais cada vez mais qualificados, fazem com que o homem seja estimulado à realização de constantes atualizações de seu conhecimento. O ensino online vem ao encontro dessa realidade e é uma ferramenta alternativa para capacitação de enfermeiros ${ }^{1-5}$.

As organizações têm utilizado o ensino online para desenvolvimento de estratégias de atualização contínua de competências organizacionais e individuais, por possibilitarem o alcance maior de pessoas do que em cursos presenciais ${ }^{6}$. Diante da evolução tecnológica e das exigências de atualização profissional permanente, o ensino de enfermagem não pode prescindir de novas tecnologias para a capacitação profissional.

A utilização de ensino online pode ser uma estratégia eficiente no processo ensino aprendizagem, porém o desenvolvimento dessa ferramenta necessita, além da perspectiva pedagógica interativa e dinâmica, de uma interface bem planejada e que seja intuitiva ao usuário ${ }^{7}$.
A andragogia tem sido adotada como caminho educacional exitoso para capacitação online dos profissionais da enfermagem, por compreender e promover o aprendizado dos adultos, tendo como base a formação na experiência que esse traz na sua história de vida, sendo essa advinda de uma formação formal ou informal ${ }^{8}$. O modelo andragógico é baseado nos pressupostos da necessidade do saber, prontidão para aprender, experiências vividas pelos aprendizes ao longo da vida (adultos acumulam mais experiências comparativamente aos jovens), orientação para aprendizagem e motivação. O adulto necessita saber o porquê de aprender um assunto novo, de correlacionar e de refletir sobre suas experiências anteriores e entender como aplicá-las em situações futuras com excelência, qualidade e eficiência. Dessa maneira, sente-se motivado ao perceber que o novo aprendizado poderá auxiliar na execução de atividades ou enfrentamento de problemas?. Nessa perspectiva, o modelo andragógico e a educação permanente na enfermagem podem ser utilizados em 
propostas educacionais online em enfermagem, visando à capacitação e à formação profissionais ${ }^{10}$.

Para a estomaterapia, como especialidade eminentemente clínica que envolve as áreas de cuidado às pessoas com estomias, feridas e incontinências, o ensino online embasado nas melhores evidências científicas tem demonstrado ser uma estratégia efetiva para atualização e capacitação dos profissionais de enfermagem e, por conseguinte, para a qualidade da assistência em saúde ${ }^{2,11,12}$.

$\mathrm{Na}$ enfermagem em estomaterapia e em dermatologia, bem como em saúde pública, as úlceras venosas (UVs) constituem sério problema, com prevalências variando de 0,18-5,69\% e incidências mais elevadas em idosos com mais de 65 anos, comprometendo a produtividade e a qualidade de vida das pessoas e famílias, além de gastos onerosos com a saúde. Além do diagnóstico e tratamento adequados serem fundamentais para o cuidado, uma das medidas preventivas e terapêuticas mais importantes é a terapia compressiva (TC), por meio da qual os pacientes podem atingir completa cicatrização em 40 a $95 \%$ dos casos. A TC consiste na aplicação de pressão à extremidade inferior das pernas como meio de facilitação do retorno venoso ao coração, e pode ser realizada com o uso de meias elásticas de compressão, sistemas de bandagens de compressão e compressão pneumática intermitente ${ }^{13,14}$.

$\mathrm{Na}$ prática clínica diária, as dificuldades encontradas pela maioria dos enfermeiros no que concerne à indicação, à aplicação e ao manuseio dos diferentes sistemas de compressão motivaram o desenvolvimento do curso específico sobre UV e TC por enfermeiros do XVII Curso de Especialização em Enfermagem em Estomaterapia da Escola de Enfermagem da Universidade de São Paulo (EEUSP), em 2009. O curso foi validado por especialistas em estomaterapia e em educação a distância ${ }^{2}$.

Os objetivos desse estudo foram avaliar o grau de conhecimento de enfermeiros sobre UV e TC; comparar o grau de conhecimento sobre a temática entre enfermeiros com e sem formação especializada em estomaterapia após a realização de curso online; e analisar as variáveis demográficas e educacionais associadas à aprendizagem.

\section{MÉTODOS}

Trata-se de estudo quase experimental e comparativo em que se testou uma intervenção didática em dois grupos distintos de enfermeiros. Foi realizado após aprovação pelo Comitê de Ética em Pesquisa (protocolo 1062/2011/CEPSISNEP CAAE: 0068.0.196.000-11).

A amostra do estudo foi constituída de 57 enfermeiros distribuídos em dois grupos: Grupo A, composto de 28 enfermeiros que cursaram especialização em enfermagem em estomaterapia na EEUSP, em 2011 e 2013; e Grupo B, composto de 29 enfermeiros generalistas que trabalhavam em oito hospitais gerais de médio e grande porte da cidade de São Paulo, em 2013. Salienta-se que a amostragem foi não probabilística por conveniência, não sendo realizado cálculo amostral.

Os enfermeiros do Grupo A foram convidados a participar da pesquisa ainda enquanto especializandos em estomaterapia e todos os componentes de ambas as turmas do curso de especialização (oferecido bienalmente) aceitaram participar da pesquisa. A seleção dos enfermeiros do Grupo B aconteceu por meio de carta-convite enviada eletronicamente aos coordenadores dos serviços de educação continuada de oito hospitais gerais da cidade de São Paulo da qual constavam os objetivos da pesquisa e os critérios de inclusão para composição do grupo: não ter formação especializada em estomaterapia e/ou dermatologia e não participar de comissões ou grupos de tratamento de feridas nas instituições de trabalho. Dessa maneira, a listagem dos interessados foi enviada pelos próprios diretores dos serviços de educação continuada, cabendo às pesquisadoras tão somente confirmar o atendimento aos critérios de inclusão. Todos os enfermeiros que compuseram a amostra do presente estudo aceitaram participar voluntariamente e sabiam que o curso era gratuito e que incluiria duas participações presenciais (no início e término do curso). Todos os participantes de ambos os grupos assinaram o Termo de Consentimento Livre e Esclarecido (TCLE) em duas vias.

Os participantes do Grupo A foram orientados pelas pesquisadoras com relação ao curso online, à navegação na plataforma Moodle e aos procedimentos da pesquisa durante o último mês do período de realização das aulas teóricas de cada edição do Curso de Especialização em Estomaterapia (em 2011 e 2013) - após o módulo teórico de cuidado a pacientes com feridas agudas e crônicas - em um encontro presencial realizado no Laboratório de Informática da EEUSP. Nessa oportunidade, os alunos assinaram o TCLE e responderam ao questionário de avaliação pré-curso (préteste). Os enfermeiros do Grupo B também participaram de um encontro presencial no mesmo local, receberam as 
mesmas instruções pelas pesquisadoras, assinaram o TCLE e fizeram o preenchimento do questionário, em 2013, em período diferente dos enfermeiros do Grupo A. Todos os enfermeiros que compuseram a amostra desta pesquisa acessaram o conteúdo do curso online e realizaram as atividades eletrônicas previstas durante um período máximo de 30 dias (a partir do encontro presencial), em horários e locais de própria escolha. Além disso, participaram de um segundo encontro realizado na EEUSP, para preenchimento do questionário de avaliação pós-curso (pós-teste), em data pré-agendada para cada grupo e após o término do período de 30 dias de atividades do curso online. Cada encontro presencial (pré e pós-curso) teve a duração de aproximadamente 1 hora e meia.

O curso online sobre UV e TC encontrava-se disponível na plataforma Moodle, no endereço eletrônico http://www. moodle.redealuno.usp.br, ao qual os participantes tiveram acesso após autorização eletrônica dos tutores por meio de cadastro realizado com nome de usuário e senha. $\mathrm{O}$ curso

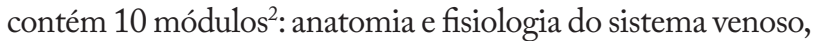
insuficiência venosa crônica, $U V$, avaliação do paciente com UV, prevenção e tratamento da UV, introdução sobre TC, bandagens, meias elásticas, compressão pneumática e estudo de caso clínico. Todos os módulos contam com: textos de apoio e diapositivos baseados nas principais referências bibliográficas sobre o assunto; literatura complementar com artigos científicos, consensos, revisões Cochrane e links para os principais sites e guidelines; e ilustrações e vídeos exemplificando o conteúdo. Ao fim de cada módulo, os enfermeiros realizaram avaliações formativas e preencheram o glossário com terminologias aprendidas. O tempo médio estimado para que o participante concluísse todos os módulos do curso foi de 20 horas, sendo 2 horas para cada módulo.

Os enfermeiros de ambos os grupos foram acompanhados por oito tutores que se dividiram com a finalidade de oferecer suporte contínuo aos participantes, para dirimir suas dúvidas e estimular seu envolvimento com o curso e com o próprio aprendizado. A tutoria decorreu de modo síncrono e assíncrono, nos quais os tutores respondiam às dúvidas postadas nos fóruns e em chat. Ao final do curso, a discussão do estudo de caso foi realizada, via chat, visando à interação, ao desenvolvimento do senso crítico e à colaboração entre enfermeiros. Os arquivos foram enviados para avaliação e obtenção da nota final do curso.

Para coleta de dados, utilizaram-se dois instrumentos. $\mathrm{O}$ primeiro permitiu o levantamento do perfil dos participantes, incluindo: idade, sexo, ano de conclusão da graduação, especialização em estomaterapia, área de atuação e participação em cursos de atualização relacionados a feridas nos últimos 5 anos. O segundo instrumento - questionário de avaliação de conhecimento sobre o tema - continha 10 questões de múltipla escolha e visou à avaliação do conhecimento dos enfermeiros sobre UV e TC (anatomia, fisiopatologia, epidemiologia, prevenção, diagnóstico e tratamento). A pontuação do questionário foi distribuída didaticamente: as questões de múltipla escolha valendo um ponto e cada sentença acertada das questões do tipo verdadeiro ou falso com valor de 0,25 , totalizando um ponto por questão. $\mathrm{O}$ valor total do teste foi de 10 pontos. O período total para a intervenção e coleta de dados foi de 31 dias para ambos os grupos, sendo 2 dias para os encontros presenciais (nos quais foram aplicados os instrumentos de coleta de dados pré- e pós-curso) e 30 dias para acesso ao curso online e desenvolvimento das atividades pelos participantes.

Os dados obtidos foram inseridos em banco específico, utilizando-se o programa Microsoft $\mathrm{Excel}^{\circledR}$, sendo posteriormente submetidos à dupla checagem para maior segurança e prevenção de erros. Consideraram-se o grau de conhecimento como variável dependente e a formação prévia, a área de atuação, a idade e o tempo de formação como variáveis independentes. A análise dos dados foi iniciada testando-se a evolução nos acertos, questão por questão, pelo teste de McNemar. A comparação dos acertos entre os grupos (A e B) foi realizada pelo teste qui-quadrado. Para modelar a evolução da nota segundo variáveis explicativas, inicialmente compararam-se as médias das notas segundo o teste F da ANOVA (3 ou mais fatores) ou teste $t$ de Student ( 2 fatores). Por fim, ajustou-se um modelo marginal utilizando equações de estimação generalizada (EEG). Para as análises, utilizou-se nível de significância de 5\%, e os resultados foram obtidos com auxílio do software estatístico R-2 15.3. ${ }^{{ }^{115} \text {. }}$

\section{RESULTADOS}

A amostra total foi constituída predominantemente por mulheres (apenas dois homens) com idades entre 22 e 53 anos, distribuindo-se homogeneamente entre os grupos quanto às faixas etárias. Aproximadamente $88 \%$ dos enfermeiros atuavam na assistência em unidades de clínica médica e cirúrgica, ambulatórios, unidades de terapia intensiva e de pronto-socorro; 30 enfermeiros $(52,6 \%)$ graduaram-se entre 
os anos de 2007 e 2012; 21 (36,8\%) entre 2000 e 2006 e apenas seis (10,5\%) formaram-se antes de 1999. Trinta e sete $(67,3 \%)$ tinham especialização em outras áreas e 35 $(62,5 \%)$ realizaram algum curso relacionado às feridas nos últimos 5 anos.

A Fig. 1 mostra que os enfermeiros do Grupo B têm menor conhecimento e domínio prévios sobre o tema comparativamente aos do Grupo A, visto que a nota mais alta apresentada pelos primeiros, antes do curso, praticamente se equipara à nota mais baixa apresentada pelo Grupo A no mesmo momento. No entanto, após o curso, ambos os grupos obtiveram médias superiores, significando ganho de conhecimento. As médias dos grupos A e B foram 7,6 e 6,3 antes do curso, respectivamente, e 9,0 e 8,5 após o curso, respectivamente. Verifica-se, ainda, que o Grupo A obteve cerca de 1,5 a mais na média final em relação à inicial, enquanto o Grupo B obteve cerca de dois pontos a mais.

A Tabela 1 mostra que houve aumento de acertos para todas as questões na amostra total. No entanto, somente para os itens anatomia e fisiologia vascular (questões 1 e 2), diferenças entre UV e úlcera arterial (UA) (questão 5), cuidados com a pele para prevenção de UV (questão 6a), TC e índice tornozelo-braço (ITB) (questão 9) e tipos de bandagens (questão 10), as diferenças foram estatisticamente significativas $(\mathrm{p}<0,05)$. Todos os enfermeiros alcançaram $100 \%$ de acertos nas questões sobre prevalência (questão 3b), avaliação - incluindo o ITB (questão 8d) - características da UV (questão 4a) e prevenção da UV (questão 6b). Percebe-se que, na amostra total e em ambos os momentos, as questões sobre anatomia e fisiologia venosa (questão 2), indicação de meias elásticas (questões 8a e 8b), definição de UA (questões 5 c e 5d) e ITB (questão 9) apresentaram menores índices de acertos em comparação às demais questões. Quanto à formação complementar prévia sobre feridas, verificou-se que $24(88,9 \%)$ enfermeiros do Grupo A realizaram, pelo menos, um curso nos últimos 5 anos, o que aconteceu para apenas 11 (37,9\%) enfermeiros do Grupo B.

Observa-se, ainda, que os enfermeiros com até 29 anos elevaram a nota em 1,89; os de 30 a 39 anos elevaram a nota em 3,02 e os com mais de 40 anos elevaram a nota em até 3,74.

Ao ser comprovada a inexistência de interações entre as faixas etárias e os conhecimentos específicos [modelo marginal inicial estimado por EEG], as correlações foram testadas entre as variáveis: faixa etária, grupo e momento, antes e após o curso, ou seja, as variações de idade e conhecimentos específicos, independentemente. A Tabela 2 apresenta as estimativas do modelo marginal final sem os componentes não significativos do modelo inicial.

O crescimento do grau de conhecimento entre os enfermeiros com idades entre 30 e 39 anos ( $p=0,028)$ ou de 40 anos ou mais $(p=0,043)$ é cerca de 0,86 superior ao ganho obtido por aqueles com idade até 29 anos. A evolução do conhecimento para o Grupo A é 0,786 ( $p=0,022)$, inferior à evolução daqueles pertencentes ao Grupo B.

Ao realizarem-se comparações múltiplas entre os grupos e idades antes e depois do curso, independentemente dos conhecimentos prévios, as notas médias obtidas no Grupo A, em ambos os momentos, são significativamente superiores àquelas alcançadas no Grupo $\mathrm{B}$, conforme mostra a Tabela 3.

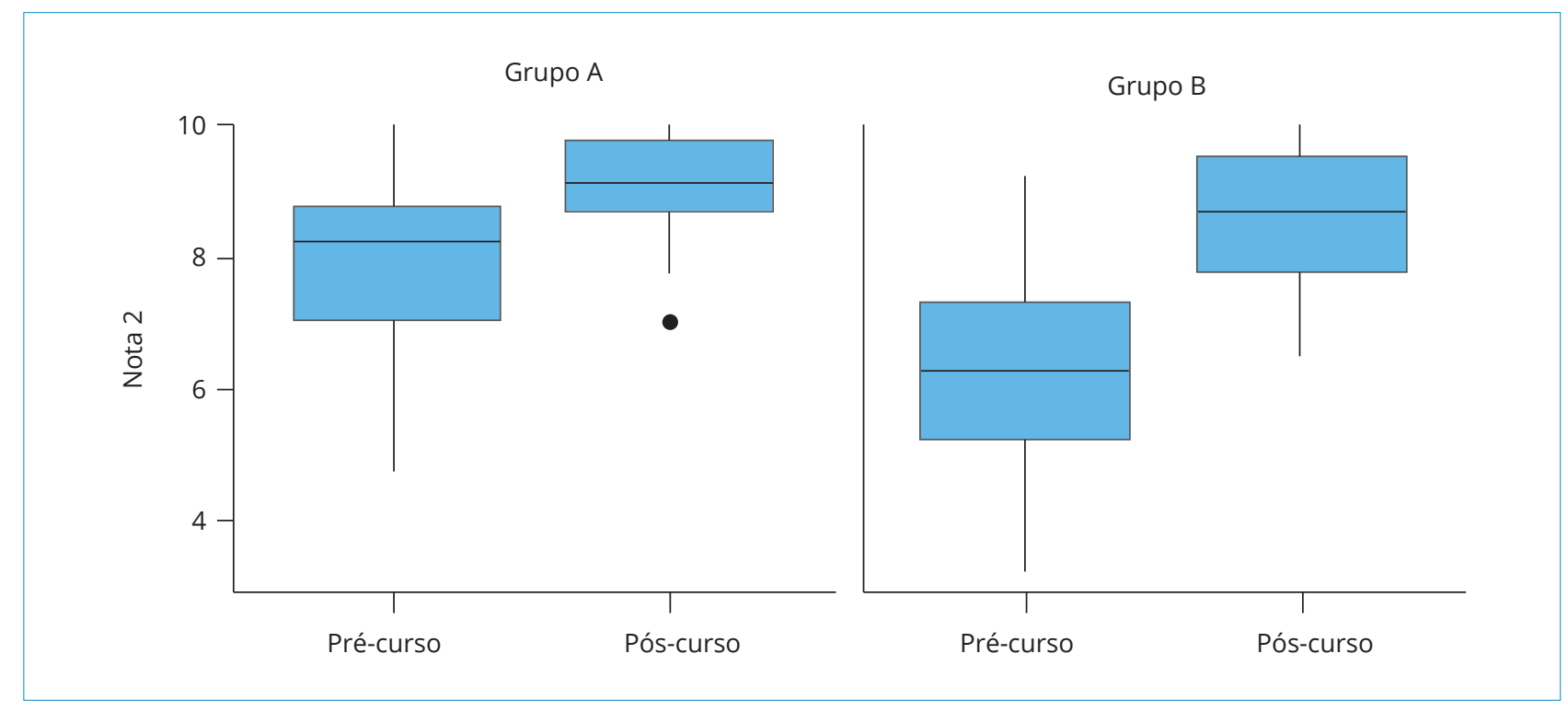

Figura 1. Médias das notas dos grupos A e B de acordo com o momento (pré- e pós-curso). São Paulo/SP, 2014. 
Tabela 1. Frequência de acertos por questão pré- e pós-curso. São Paulo/SP, 2014.

\begin{tabular}{|c|c|c|c|c|c|}
\hline \multirow[t]{2}{*}{ Questão } & Acerto antes & Acerto após & $\begin{array}{l}\text { Erro antes, } \\
\text { acerto após }\end{array}$ & $\begin{array}{c}\text { Acerto antes, } \\
\text { erro após }\end{array}$ & p-valor* \\
\hline & n (\%) & $\mathrm{n}(\%)$ & $\mathrm{n}(\%)$ & n (\%) & \\
\hline 1 (Anatomia) & $29(50,8)$ & $52(91,2)$ & $23(40,4)$ & - & $<0,001$ \\
\hline 2 (Anatomia e fisiologia) & $18(31,6)$ & $42(73,7)$ & $30(52,6)$ & $6(10,5)$ & $<0,001$ \\
\hline 3a (Definição de UV) & $51(89,4)$ & $56(98,2)$ & $5(8,8)$ & - & 0,074 \\
\hline 3b (Prevalência) & $55(96,5)$ & $57(100,0)$ & $2(3,5)$ & - & 0,48 \\
\hline 3c (Fisiopatologia de UV) & $51(89,5)$ & $56(98,2)$ & $6(10,5)$ & 1,8 & 0,131 \\
\hline 3d (Fisiopatologia de UV) & $49(85,9)$ & $54(94,7)$ & $7(12,3)$ & $2(3,5)$ & 0,182 \\
\hline $4 a$ (Características de UV) & $55(96,5)$ & $57(100,0)$ & $2(3,5)$ & - & 0,48 \\
\hline 4b (Características de UV) & $54(94,7)$ & $55(96,5)$ & $3(5,3)$ & $2(3,5)$ & 1,00 \\
\hline 4c (Características de UV) & $50(87,7)$ & $52(91,2)$ & $6(10,5)$ & $4(7,0)$ & 0,752 \\
\hline 4d (Características de UV) & $51(89,4)$ & $53(92,9)$ & $5(8,8)$ & $3(5,3)$ & 0,724 \\
\hline 5a (Características de UA) & $43(75,4)$ & $53(93,0)$ & $12(21,1)$ & $2(3,5)$ & 0,016 \\
\hline 5b (Características de UV) & $50(87,7)$ & $55(96,4)$ & $6(10,5)$ & $1(1,8)$ & 0,131 \\
\hline $5 \mathrm{c}$ (Características de UA) & $41(71,9)$ & $45(78,9)$ & $9(15,8)$ & $5(8,8)$ & 0,423 \\
\hline $5 \mathrm{~d}$ (Características de UV/UA) & $52(91,2)$ & $51(89,4)$ & $4(7,0)$ & $5(8,8)$ & 1,00 \\
\hline 6a (Prevenção de UV) & $33(57,9)$ & $50(87,7)$ & $18(31,6)$ & $1(1,8)$ & $<0,001$ \\
\hline 6b (Prevenção de UV) & $57(100,0)$ & $57(100,0)$ & - & - & - \\
\hline 6c (Prevenção de UV) & $50(87,7)$ & $49(86,0)$ & $5(8,8)$ & $6(10,5)$ & 1,00 \\
\hline 6d (Prevenção de UV) & $52(91,2)$ & $56(98,2)$ & $4(7,0)$ & - & 0,134 \\
\hline 7 (TC e coberturas) & $50(87,7)$ & $51(89,5)$ & $5(8,8)$ & $4(7,0)$ & 1,00 \\
\hline 8a (Meias elásticas) & $39(68,4)$ & $46(80,7)$ & $13(22,8)$ & $6(10,5)$ & 0,169 \\
\hline $8 \mathrm{~b}$ (Meias elásticas) & $25(43,9)$ & $32(56,2)$ & $13(22,8)$ & $6(10,5)$ & 0,169 \\
\hline 8c (Avaliação de UV-ITB) & $44(77,2)$ & $52(91,2)$ & $11(19,3)$ & $3(5,3)$ & 0,061 \\
\hline 8d (Avaliação de UV-ITB) & $53(93,0)$ & $57(100,0)$ & $4(7,0)$ & - & 0,134 \\
\hline 9 (ITB e TC) & $20(35,1)$ & $37(64,9)$ & $24(42,1)$ & $7(12,3)$ & 0,004 \\
\hline 10 (TC e bandagens) & $44(77,1)$ & $56(98,2)$ & $12(21,1)$ & - & 0,001 \\
\hline
\end{tabular}

UA = úlcera arterial; UV = úlcera venosa. * Teste McNemar. ITB = índice tornozelo-braço; TC = terapia compressiva;

Tabela 2. Parâmetros estimados para o modelo marginal final. São Paulo/SP, 2014.

\begin{tabular}{|c|c|c|c|}
\hline Coeficientes & Estimado & Erro padrão & p-valor \\
\hline Intercepto & 7,238 & 0,272 & $<0,001$ \\
\hline Idade (30-39) & $-1,207$ & 0,327 & $<0,001$ \\
\hline Idade (40 ou mais) & $-2,034$ & 0,458 & $<0,001$ \\
\hline $\begin{array}{l}\text { Conhecimentos } \\
\text { específicos (Grupo A) }\end{array}$ & 1,376 & 0,305 & $<0,001$ \\
\hline Tempo (pós-curso) & 1,596 & 0,336 & $<0,001$ \\
\hline $\begin{array}{l}\text { Idade (30-39) - Tempo } \\
\text { (pós-curso) }\end{array}$ & 0,870 & 0,395 & 0,028 \\
\hline $\begin{array}{l}\text { Idade (40 ou mais) - } \\
\text { Tempo (pós-curso) }\end{array}$ & 0,856 & 0,423 & 0,043 \\
\hline $\begin{array}{l}\text { Conhecimentos } \\
\text { específicos (Grupo A) - } \\
\text { Tempo (pós-curso) }\end{array}$ & $-0,786$ & 0,342 & 0,022 \\
\hline Parâmetro de escala & 1,195 & 0,174 & $<0,001$ \\
\hline $\begin{array}{l}\text { Parâmetro de } \\
\text { correlação (pré-pós) }\end{array}$ & 0,284 & 0,105 & 0,007 \\
\hline
\end{tabular}

Tabela 3. Comparações múltiplas baseadas no modelo marginal final. São Paulo/SP, 2014.

\begin{tabular}{|c|c|c|c|}
\hline Comparações múltiplas & Estimativa & Erro padrão & p-valor \\
\hline \multicolumn{4}{|l|}{ Antes do curso } \\
\hline $\begin{array}{l}\text { Idade } 20 \text { a } 29 \\
\text { contra } 30 \text { a } 39 \text { anos }\end{array}$ & 1,207 & 0,327 & $<0,001$ \\
\hline $\begin{array}{l}\text { Idade } 20 \text { a } 29 \\
\text { contra } 40 \text { anos ou mais }\end{array}$ & 2,034 & 0,458 & $<0,001$ \\
\hline $\begin{array}{l}\text { Idade } 30 \text { a } 39 \\
\text { contra } 40 \text { anos ou mais }\end{array}$ & 0,827 & 0,413 & 0,045 \\
\hline Grupo A contra Grupo B & 1,376 & 0,305 & $<0,001$ \\
\hline \multicolumn{4}{|l|}{ Depois do curso } \\
\hline $\begin{array}{l}\text { Idade } 20 \text { a } 29 \\
\text { contra } 30 \text { a } 39 \text { anos }\end{array}$ & 0,337 & 0,335 & 0,314 \\
\hline $\begin{array}{l}\text { Idade } 20 \text { a } 29 \\
\text { contra } 40 \text { anos ou mais }\end{array}$ & 1,179 & 0,419 & 0,005 \\
\hline $\begin{array}{l}\text { Idade } 30 \text { a } 39 \\
\text { contra } 40 \text { anos ou mais }\end{array}$ & 0,841 & 0,413 & 0,042 \\
\hline Grupo A contra Grupo B & 0,590 & 0,301 & 0,050 \\
\hline
\end{tabular}




\section{DISCUSSÃO}

A relevância de um curso online sobre o cuidado de pessoas com UVs com enfoque em TC pode ser considerada no sentido de agregar conhecimentos específicos aos profissionais de enfermagem de maneira flexível e inovadora, por meio de tecnologias educacionais. A educação a distância online tem características que permitem flexibilidade de horários e de deslocamentos físicos que facilitam a capacitação do enfermeiro. Certamente, conhecimentos sobre a temática permitem que os profissionais atuem na prevenção e tratamento da UV, como uma ação do cuidar baseado em evidências, essência da assistência da enfermagem. Nesse sentido, para o sucesso da aprendizagem dos profissionais da área da saúde,é importante o uso de estratégias inovadoras tal como o curso online sobre UV - adequadas aos conteúdos e aos objetivos que permitam o desenvolvimento de habilidades e incorporação de valores ${ }^{16}$.

O curso online sobre UV permitiu a aprendizagem sobre intervenções de enfermagem e contribuiu para o aprofundamento das bases teóricas que fundamentam ações e decisões por meio do raciocínio clínico. Nesse sentido, os cursos online estimulam a autoconfiança e o compartilhamento de experiências, bem como promovem ambiente de respeito mútuo e aprendizagem estruturada e individualizada ${ }^{16}$.

Outro fator a ser considerado refere-se à busca pelo curso. $\mathrm{O}$ interesse dos enfermeiros generalistas pelo tema motivou-os a buscar o aprimoramento quando foi realizado o convite aos profissionais dos hospitais. Dessa forma, reforça-se a necessidade de abordar assuntos que vão ao encontro dos anseios daqueles que desejam aprender, despertando, assim, o interesse, a motivação e a elucidação de dúvidas.

Os resultados deste estudo mostraram que, após a realização do curso online Úlcera Venosa com Enfoque em Terapia Compressiva, houve crescente apreensão do conhecimento por todos os enfermeiros da amostra. Observou-se aumento significativo na nota (grau de conhecimento) para aqueles com idade igual ou superior a 30 anos, com maior relevância para aqueles com idade igual ou superior a 40 anos.

Analisando-se a influência da idade no desempenho dos enfermeiros, ou seja, na avaliação do grau de conhecimento obtido após o curso Úlcera Venosa com Enfoque em Terapia Compressiva, verificou-se que, em ambos os grupos, quanto menor a idade, maior a nota média após o curso. No entanto, apesar de os mais jovens apresentarem médias mais elevadas, os participantes que tinham 40 anos ou mais obtiveram melhor aproveitamento no curso, ou seja, foi o grupo que mais elevou a média em relação ao grau de conhecimento após o curso. De acordo com o modelo andragógico, as experiências vivenciadas ajudam no processo de aprendizagem, sendo que adultos aprendem mais no contexto da vida real, sendo motivados a aprender para solucionar problemas $^{10,17}$. À medida que as pessoas amadurecem, passam a ser independentes, a acumular experiências de vida que fundamentam seu aprendizado, a direcionar seus interesses para o desenvolvimento das habilidades, a esperar pela aplicação prática do que aprendem e a aprender para resolver problemas e desafios ${ }^{10}$.

Os resultados encontrados mostram, portanto, a relação entre a idade e o desempenho dos participantes de cursos online e apontam para a necessidade de adoção de estratégias de ensino específicas para cada estágio da vida. Alunos enfermeiros com maior grau de autonomia podem apresentar melhor desempenho, e o aprendizado ocorre pelo grau de responsabilidade que se assume em relação às atividades. Pesquisa evidencia que o ensino online favorece ao estudante a conscientização sobre a própria performance de atuação em atendimento de emergência e permite o acesso ao conhecimento, atuando como espaço de saber e ambiente de reflexão, estimulando o raciocínio clínico e a tomada de decisão em enfermagem ${ }^{18}$.

Existe uma gama de estudos que evidenciam a existência de características preditoras de melhor desempenho em cursos a distância relacionadas com idade, existência de experiências anteriores, interação, autocontrole, disciplina e capacidade de comunicação ${ }^{19}$. Embora não constem dos objetivos do estudo, é importante mencionar que os enfermeiros de ambos os grupos avaliaram o material didático e a tutoria do curso online como estratégias adequadas para o alcance de seus objetivos, ou seja, a melhora do grau de conhecimento. Os tutores do curso online assumiram o papel de mediadores da aprendizagem, o que está de acordo com a literatura, pois o tutor deve motivar e despertar o interesse dos participantes, considerando o contexto em que a aprendizagem será aplicada e como cada um será tutorado ${ }^{20}$.

Os desafios do ensino online não são apenas de ordem instrumental, mas, também, da postura que o meio educacional 


\section{CONCLUSÃO}

deve ter frente às inovações tecnológicas como componente pedagógico eficiente e libertador para que não se tornem apenas replicadoras de informação, mas meios de socialização de conhecimento e campo de práticas promotoras de novas possibilidades educacionais mediadas, para contribuir com a formação de indivíduos críticos e criativos ${ }^{21}$.

As limitações deste estudo referem-se ao processo de amostragem não probabilística de conveniência e à ausência de grupo-controle que, embora dificultem generalizações, permitem obter uma boa imagem do universo estudado e indicar que existe relação entre variáveis demográficas e educacionais associadas à aprendizagem em cursos online. Destacam-se, no entanto, contribuições por se tratar de estudo de intervenção, tão necessário para a ampliação do corpo de evidências em enfermagem. Além disso, emprega e analisa moderna ferramenta educacional baseada na andragogia, cuja utilização deve ser ampliada, maximizada e otimizada, principalmente ao se considerarem as carências profissionais na área do cuidar de pessoas com feridas em um país de dimensão continental e áreas remotas, como é o Brasil.

É pertinente aprofundar e ampliar o conhecimento nessa área por meio de pesquisas experimentais, para possibilitar a análise de relações causais entre as variáveis, bem como a avaliação dos cursos presenciais ou online e o impacto na assistência à saúde.
Os resultados favoráveis obtidos no presente estudo de avaliação do curso online sobre UV e TC, junto aos enfermeiros com e sem formação especializada, permitem que sejam recomendadas sua divulgação e implementação para equipes de enfermagem e multidisciplinares em diferentes cenários de atenção à saúde, desde que os conteúdos e objetivos educacionais sejam adaptados ao público-alvo.

Os resultados do estudo confirmam que houve aquisição de conhecimento sobre UV e TC entre os enfermeiros com e sem formação especializada em estomaterapia e que os enfermeiros especializados desta amostra detêm conhecimento maior em relação ao tema quando comparados aos enfermeiros não especializados, principalmente no momento pré-curso online.

\section{CONTRIBUIÇÃO DOS AUTORES}

Conceitualização, Schmidt FMQ; Aroldi JB; Peres HHC; Quiroz LM; dos Anjos PP; Teixeira VA e Santos VLCG; Metodologia, Schmidt FMQ; Aroldi JB; Peres HHC e Santos VLCG; Redação, Schmidt FMQ; Aroldi JB; Quiroz LM; dos Anjos PP e Teixeira VA; Supervisão, Peres HHC e Santos VLCG.

\section{REFERÊNCIAS}

1. Figueiredo MA, Amaral RCB. Planejamento e gestão das disciplinas na modalidade a distância em cursos de graduação presencial: conteúdo, aprendizagem e construção do conhecimento. Ciência Atual. 2013;1:23-31.

2. Queiroz FM, Aroldi JBC, Oliveira GDS, Peres HHC, Santos VLCG. Úlcera venosa e terapia compressiva para enfermeiros: desenvolvimento de curso online. Acta Paul Enferm 2012;25(3):435-40.

3. Colella $\mathrm{CL}$, Beery TA. Teaching differential diagnosis to nurse practitioner students in a distance program. J Nurs Educ. 2014;53(8):433-8. https://doi.org/10.3928/0148483420140724-02

4. Hodges AL, McGuinness T. Improving communication in distance-accessible advanced practice nursing courses via instructor presence. J Nurs Educ. 2014;53(8):479-82. https:// doi.org/10.3928/01484834-20140725-03

5. Shillam CR, Ho G, Commodore-Mensah Y. Online biostatistics: evidence-based curriculum for master's nursing education. J Nurs Educ. 2014;53(4):229-32. https:// doi.org/10.3928/01484834-20140326-01
6. Zerbini T, Abbad G. Qualificação profissional a distância: avaliação da transferência de treinamento. Paidéia. 2010;20(47):313-23.

7. Rodrigues RCV, Peres HHC. An educational software development proposal for nursing in neonatal cardiopulmonary resuscitation. Rev Esc Enferm USP. 2013;47(1):231-7. https:// doi.org/10.1590/s0080-62342013000100030

8. Knowles MS, Swanson RA, Holton EF. The adult learner: the definitive classic in adult education and human resource development. 6a ed. San Diego: Elsevier Science and Technology Books; 2005.

9. Knowles MS, Holton III EF, Swanson RA. Aprendizagem de resultados: uma abordagem prática para aumentar a efetividade da educação corporativa. Rio de Janeiro: Elsevier; 2009.

10. Draganov PB, Friedländer MR, Sanna MC. Andragogia na saúde: estudo bibliométrico. Escola Anna Nery. 2011;15(1):149-56.

11. Gonçalves MBB, Rabeh SAN, Terçario CAS. Contribuição da educação a distância para o conhecimento de docentes 
de enfermagem sobre avaliação de feridas crônicas. Rev Latino-Am Enfermagem. 2015;23(1):122-9. https://doi.org/ 10.1590/0104-1169.3606.2533

12. Rabeh S, Gonçalves MBB, Caliri MHL, Nogueira PC, Miyazak MY. Terapia tópica para heridas crónicas: contribuciones de un módulo de enseñanza a distancia para el conocimiento de estudiantes de enfermeira. Enfermería Global. 2017;16(1):8090. https://doi.org/10.6018/eglobal.16.1.237361

13. O'Meara S, Cullum NA, Nelson EA, Dumville JC. Compression for venous leg ulcers. Cochrane Database Syst Rev. 2012;(11):CD000265. https://doi.org/10.1002/14651858. cd000265.pub3

14. Ratliff CR, Yates S, McNichol L, Gray M. Compression for primary prevention, treatment, and prevention of recurrence of venous leg ulcers. J Wond Ostomy Continence Nurs. 2016;43(4):34764. https://doi.org/10.1097/WON.0000000000000242

15. R Core Team. R: A language and environment for statistical computing. R Foundation for Statistical Computing. Disponível em: http://www.R-project org/

16. Draganov PB, Andrade AC, Neves VR, Sanna MC. Andragogy in nursing: a literature review. Invest Educ Enferm. 2013;31(1):8694.
17. Camargo LB, Raggio DP, Bonacina CF, Wen CL, Mendes FM, Bonecker MJ et al. Proposal of e-learning strategy to teach atraumatic restorative treatment (ART) to undergraduate and graduate students. BMC Research Notes. 2014;7:45660. https://doi.org/10.1186/1756-0500-7-456

18. Tobase L, Peres HHC, Tomazini EAS, Teodor SV, Ramos MB, Polastri TF. Suporte básico de vida: avaliação da aprendizagem com uso de simulação e dispositivos de feedback imediato. Rev Latino-Am Enferm. 2017;25:e2942. https://doi org/10.1590/1518-83451957 2942

19. Wang CH, Shannon DM, Ross ME. Students' characteristics, selfregulated learning technology self-efficacy and course outcomes in online learning distance. Distance Education. 2014;34(3):30223. https://doi.org/10.1080/015879192013 835779

20. Gould D, Papadopoulos I, Kelly D. Tutors' opinions of suitability of online learning programmes in continuing professional development for midwives. Nurse Education Today. 2014;34(Espec):613-8. https://doi.org/10.1016/jnedt 201306006

21. Fofonca $E$, Schoninger RRZV, Costa CS da. A mediação tecnológica e pedagógica em ambientes virtuais de aprendizagem: contribuições da educomunicação. Revista Tempos e Espaços em Educação. 2018;24(11):267-78. https:// doi org/10.20952/revteev11i246031 
